\title{
Microfluidic chip containing porous gradient for chemotaxis study
}

\author{
${ }^{\mathrm{a}}$ Aswan Al-Abboodi, ${ }^{\mathrm{a}}$ Ricky Tjeung, ${ }^{\mathrm{b}}$ Pauline Doran, ${ }^{\mathrm{a}}$ Leslie Yeo, ${ }^{\mathrm{a}} \mathrm{James}$ Friend, ${ }^{\mathrm{a}}$ Peggy Chan \\ ${ }^{a}$ Micro/ Nanophysics Research Laboratory, Monash University, Australia \\ ${ }^{\mathrm{b}}$ Biological science, Monash university, Australia
}

\begin{abstract}
We have developed a new porous gradient microfluidic device based on in situ Gtn-HPA/CMC-Tyr hydrogel that comprises gelatin hydroxyphenylpropionic acid (Gtn-HPA) conjugate and carboxymethyl cellulose tyramine (CMC-Tyr) conjugate. The device is fabricated using a soft lithographic technique, in which microstructures were patterned on a thin layer of polydimethylsiloxane (PDMS) using a polymeric mold. Human fibrosarcoma cells (HT1080) were employed as invasive cancer cell model. Porosity gradients were generated by flowing pore etching fluid in the gradient generator network. Results suggested that spatial control of the porosity can be obtained, which mimics the 3-dimensional microenvironment in vivo for cell-based screening applications including real time chemotaxis, cytotoxicity, and continuous drug-response monitoring. A chemoattractant gradient is then generated and cell migration is monitored in real time using fluorescence microscopy. The viability of cells was evaluated using calcien AM stain. Herein, we successfully monitored the chemotactic responses of cancer cells, confirmed the validity of using in situ porous hydrogels as a construction material for a microchemotaxis device, and demonstrated the potential of the hydrogel with tunable porosity based microfluidic device in biological experiments. This device will also be practical in controlling the chemical and mechanical properties of the surroundings during the formation of tissue engineered constructs.
\end{abstract}

Key words: porous hydrogel, microfluidic device, chemotaxis, hydrogel gradient.

\section{INTRODUCTION}

A vital part of cell biology is cell migration [1], which is regularly controlled by soluble chemical attractants or repellants (chemotaxis) as well as immobilized (haptotaxis) molecules and substrate mechanics (durotaxis) in the extracellular environment. Several studies have investigated the influence of these gradients of molecules and cues on specific cellular behavior. Additionally, these studies have investigated the effect of both chemical and physical properties of the matrix materials and shown their role in demonstrating cellular behavior in the surrounding microenvironment. Given the ability of cells to migrate toward extracellular gradient of chemicals, chemotaxis, in particular, plays an important role in many of biological and physiological processes such as creation of new tissues, wound healing, cancer metastasis and embryogenesis.

Extensive methods have been performed recently for chemotaxis assays using a variety of chemical gradients including Boyden's assays [2], Dunn's chamber [3] and micropipette-based assays [4]. All of these approaches have relied on complex and experimentally intensive techniques for gradient production, and hence have restrictions such as the involving endpoint examination, poorly controlled chemical gradients, inability to be used directly for in vivo analysis, necessity for large amounts of reagents and cell samples and inability to provide reproducible, controllable, and steady linear gradients. Hence, a diversity of microfluidic devices have been fabricated for developing simple techniques to construct model substrates that can be used to examine the complex cell behavior in vitro and to study chemotaxis in various cells types, in particular, tumour cells.

Hydrogels are networks of polymer chains that are water-swollen and can absorb considerable quantities of aqueous solutions. Due to their high water content, hydrogels are similar to natural living tissue. Thus, hydrogel have demonstrated considerable promise for drug delivery and tissue engineering [5] as a result of their controllable mechanical properties and their biocompatibility and biodegradability. As in the body, all cells are embedded in a 3D microenvironment and the ability to understanding cancer cell migration in a biomimetic $3 \mathrm{D}$ microenvironment is particularly useful in drug discovery.

Smart Nano-Micro Materials and Devices, edited by Saulius Juodkazis, Min Gu, Proc. of SPIE Vol. 8204, 82041H · @ 2011 SPIE · CCC code: 0277-786X/11/\$18 · doi: 10.1117/12.904870 
Here, we develop a hydrogel-based microfluidic chip that was specifically designed and fabricated to facilitate chemotaxis studies. The device integrates a chemical concentration gradient generator and an in situ Gtn-HPA/CMC-Tyr hydrogel with tunable porosity. The microfluidic chip consists of a microscope slide and covered PDMS film, which is designed as a chemical concentration gradient generator containing a series of cell culture chambers, microchannels, inlets and outlets. PDMS, glass and a range of hydrogel systems are generally used materials in the biomedical field. We chose primate fibrosarcoma cell line HT1080 given its relatively pure, readily generated cell population that can resemble an invasive cancer cell model. In this paper, we have investigated the possibility of studying cancer cells migration and chemotaxis using the new microfluidic gradient device. Moreover, we have demonstrated that maintaining the cell growth inside the porous hydrogel structure can offer information that can potentially be used for the development of therapies, such as scaffolding for tissue engineering. More importantly we have showed that the chemotactic movement and the behaviour of HT1080 cells in response to the chemical gradients can be successfully controlled even in the absence of stimulating factors with minimal requirements in cost or expertise.

\section{METHODOLOGY}

\subsection{Materials}

Gelatin (Gtn) $\left(\mathrm{M}_{\mathrm{w}}=80-140 \mathrm{kDa}, \mathrm{pI}=5\right)$ and horseradish peroxidase (HRP) (100 units/mg) were obtained from Wako Pure Chemical Industries (Japan). 3,4-Hydroxyphenylpropionic acid (HPA), N-hydroxysuccinimide (NHS), 1-ethyl-3-(3dimethylaminopropyl)-carbodiimide hydrochloride (EDC.HCl), carboxymethyl cellulose (CMC) $\left(\mathrm{M}_{\mathrm{w}}=90 \mathrm{kDa}\right)$ and tyramine hydrochloride (Tyr) were purchased from Sigma-Aldrich (Australia). Hydrogen peroxide $\left(\mathrm{H}_{2} \mathrm{O}_{2}\right)$ was acquired from Merck (Australia). calcein-AM and ethidium homo- dimer (LIVE/DEAD Viability/Cytotoxicity Kit, Invitrogen). Poly dimethylsiloxane (PDMS) (Sylgard 184, Dow Corning) were purchased from Ellsworth Adhesives Asia Ltd (Hongkong).

\subsection{Fabrication and assembly of the microfluidic device}

The device master mold was constructed using a 3-D printer (Eden 3D, Objet Geometries Inc., USA); the material chosen was a transparent UV curable polymeric material (Objet FullCure $720^{\mathrm{TM}}$ ). The desired patterns were designed using AutoCAD design software (Autodesk, Inc., USA). The 3-D printer was chosen due to its ability to overcome the main limitation of conventional photolithographic techniques. The mold consists of two inlet reservoirs, planar dimension of $8 \times 8 \mathrm{~mm}^{2}$ and $500 \mu \mathrm{m}$ height, one main reservoir for the reaction to occur, planar dimension of $15 \times 15$ $\mathrm{mm}^{2}$ and $300 \mu \mathrm{m}$ height, two microchannels connecting the inlet to the main reservoirs, planar dimension of $3.5 \times 0.2$ $\mathrm{mm}^{2} \mu \mathrm{m}$ and $200 \mu \mathrm{m}$ height, one microchannel for each of the inlet reservoir for flushing purpose, planar dimension of $0.3 \times 8 \mathrm{~mm}^{2}$ and $200 \mu \mathrm{m}$ height and one microchannel for the main reservoir for flushing purpose, planar dimension of $0.3 \times 5 \mathrm{~mm}^{2}$ and $200 \mu \mathrm{m}$ height. The PDMS mold was then permanently bonded to a glass slide after surface treatment using a plasma system (Harrick Plasma, Inc.).

\subsection{Synthesizing of Gtn-HPA/CMC-Tyr hydrogel}

Gtn-HPA conjugates were prepared as described by Lee et al. [6] using a general carbodiimide/active ester-mediated coupling reaction in distilled water. The CMC-Tyr conjugates were prepared by adding carboxymethyl cellulose CMC and tyramine hydrochloride (Tyr) to milliQ water. To this solution, NHS and EDC were immediately added. The same steps for preparing Gtn-HPA were followed to obtain CMC-Tyr conjugates. The Gtn-HPA/ CMC-Tyr hydrogel was prepared by mixing 5\% (w/v) from both Gtn-HPA and CMC-Tyr conjugate solutions in a weight ratio 80:20 respectively. Then the mixture was vortexed vigorously for a few minutes. The resulting polymer could be polymerized by the enzymatic oxidative coupling reaction by adding HRP (3.86 unit/L) and diluted $\mathrm{H}_{2} \mathrm{O}_{2}$ with concentration of 49.8 $\mu \mathrm{M}$. Hydrogel/ cell mixture and hydrogel free cells were injected to the main reservoir in the microfluidic device. The injected mixtures were allowed to gelate, wherein phase separation was induced by polymerization such that a polymer rich phase (a continuous phase) and a polymer poor phase (a disperse phase) was formed. Human fibrosarcoma HT1080 cell line (Sigma Aldrich, Australia) were used as model cells. 


\subsection{Chemotaxis experiments}

Immediately after fabrication of the device, $40 \mu \mathrm{l}$ of the polymerised Gtn-HPA/ CMC-Tyr hydrogel solutions with HT1080 cells were injected into the main reservoirs of the two microfluidic devices, one of them assuming the role of a control experiment. In both these devices, the right inlet reservoirs were injected with $10 \mu 1$ complete DMED medium added with FBS serum whilst the left reservoirs were injected with $10 \mu$ cellulase enzyme solution with a concentration of $0.1 \mathrm{unit} / \mathrm{ml}$, which is below the $\mathrm{LC}_{50}$ [7]. These microfluidic devices were incubated for three days in order to degrade the CMC-Tyr conjugate in Gtn-HPA/ CMC-Tyr hydrogel such that larger pore sizes are obtained. Laser scanning confocal microscopy (LSCM) images were acquired after $2 \mathrm{hrs}$ and after three days to visualise the hydrogel inside the microchannel devices. After three days, the complete medium was removed from the right inlet reservoir in the chemotaxis device and replaced with only FBS serum. At the same time the cellulase enzyme solution from the left inlet reservoirs in both the control and chemotaxis devices was removed and replaced with complete medium with FBS serum in the control device and with DMEM medium without FBS in the chemotaxis device. A Calcein AM (Invitrogen) was used to stain the cells and three separate regions (left, middle and right) from the main reservoir in each device were chosen to image the cell migration using a LSCM confocal microscope.

\section{RESULTS ND DISCUSSION}

\subsection{Fabrication and characterisation of the microfluidic device}

The soft lithographic fabrication technique, wherein PDMS was molded to form a microchannel network with three chambers, allowed encapsulation of the cells within the hydrogel matrix in a 3D structure. The development of perfusable microfluidic hydrogels and complementary photolithographic fabrication methodologies provides a robust tool set to construct complex, micro-scale, biomimetic, in vitro tissue analogues. The fluorescent light intensity emitted by the fluorescent solution of FITC- BSA in a microfluidic channel was traced using LSCM to see whether the dye will travel through the hydrogel network by diffusion.

(A)

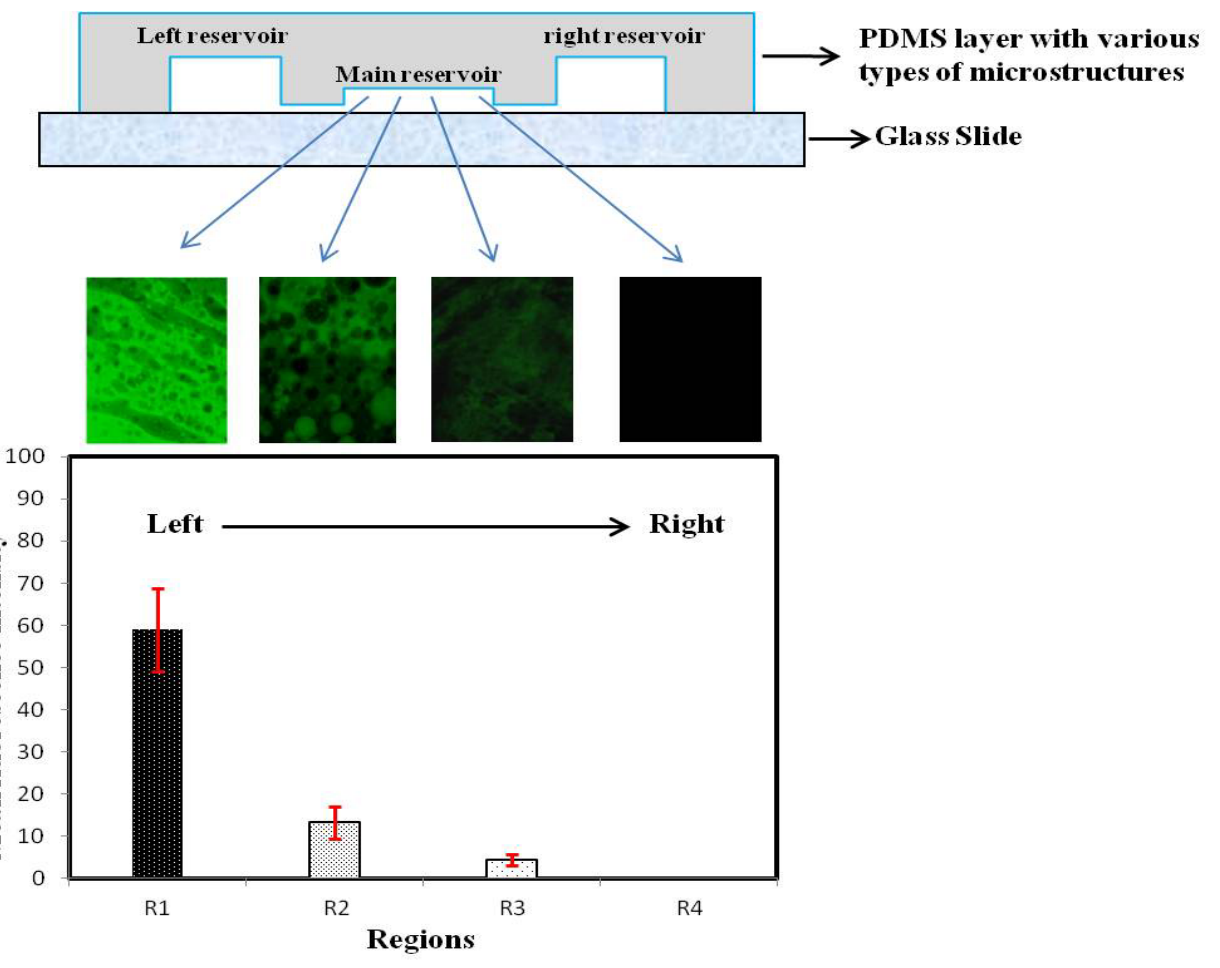

Figure 1 (A) Schematic for the device. (B) Image for each region in the main storage. (C) Average fluorescent intensity of FITC- BSA at four regions from left to right through the porous hydrogel in the main storage $\pm \mathrm{SD}$. 
Figure 1 records the fluorescent intensity of FITC- BSA in the four different regions (R1, R2, R3 and R4) from left to right in the main channel. This visual observation is further supported by the fluorescence intensity graph across these regions; this graph reflects the average intensity for six images from each region. The device was provided with enough volume of medium and chemoattractant every day during the whole time of the experiments.

\subsection{Chemotaxis experiments}

The critical issues associated with device fabrication for biological applications are its compatibility with the biological system, integrity and bio-functionality. These issues were addressed and resolved by the selection of available biocompatible materials. The FBS represents the chemoattractant material that diffuses from the right reservoir. The middle region of the device comprises an open space for hydrogel swelling due to hydration as well as for cellulase release inside the device from the left reservoir to degrade the carboxymethyl cellulose in Gtn-HPA/ CMC-Tyr hydrogel. Hydrogel was in its liquid form during the device loading after which it formed a gel in less than 3 min after adding the $\mathrm{H}_{2} \mathrm{O}_{2}$ and HRP as crosslinker materials. To form the porous gradient, as shown in Figure 2, when the cellulase enzyme was added in a concentration below the $\mathrm{LC}_{50}$ to the left reservoir, we observed through the LSCM a variation in the hydrogel pore sizes. This was related to degrade of the carboxymethyl cellose in the $5 \mathrm{wt} \% \mathrm{Gtn}-\mathrm{HPA} / \mathrm{CMC}-\mathrm{Tyr}$ hydrogel after three days. It is obvious from the images that the pore size in the region closest to the left reservoir was the largest compared with the middle and right regions. These results reflect the gradually increasing in the porosity from the left to the right regions.
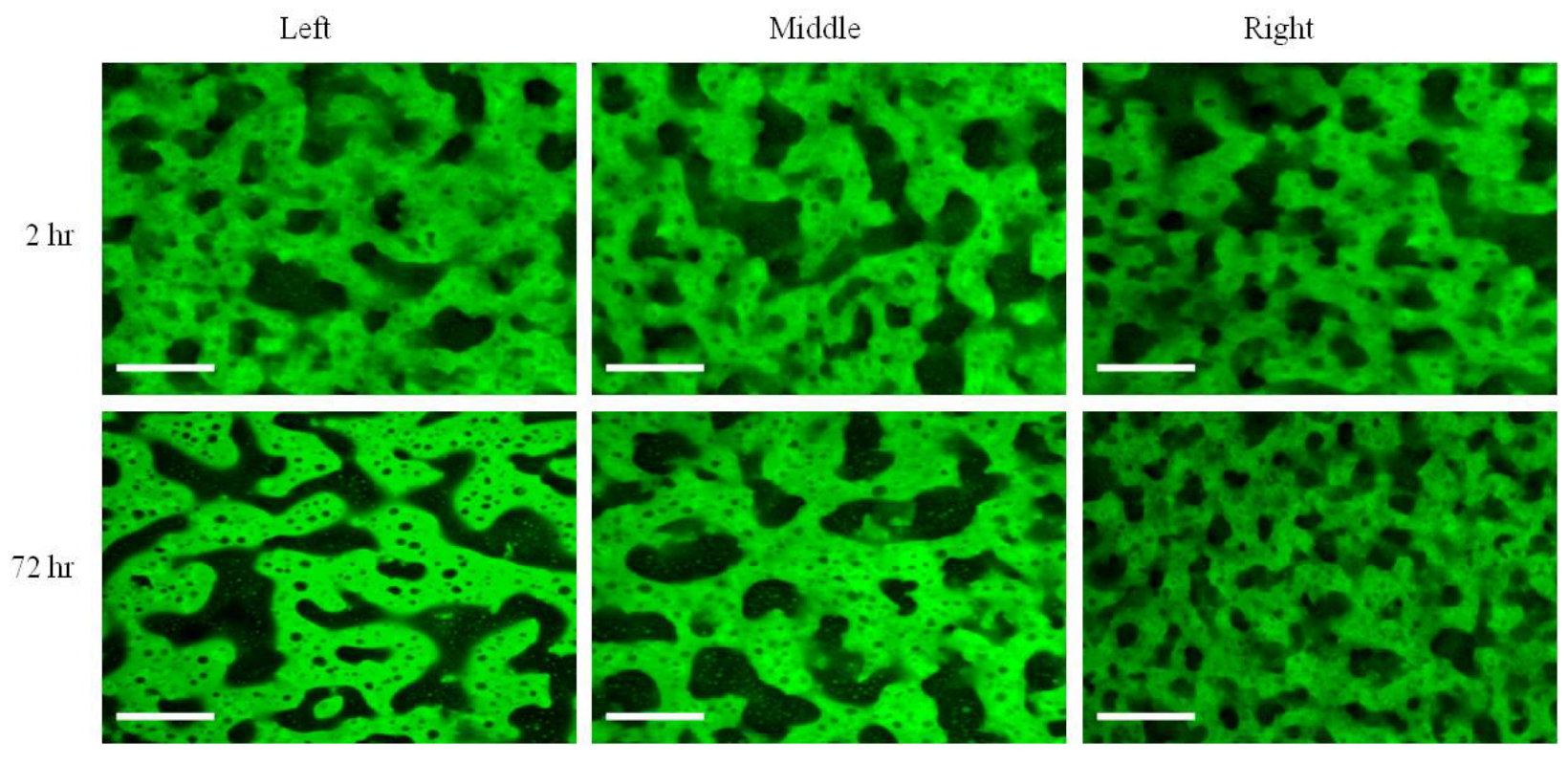

Figure 2 Three regions from the porous hydrogel (left, middle and right) in the main reservoir show the variation in the pore size after the injection of cellulase enzyme in the left reservoir. The LSCM images show the porous structure after 2 hrs (the upper images) and after $72 \mathrm{hrs}$ (the lower images). The scale bar denotes a length of $50 \mu \mathrm{m}$.

Cell migration is defined as the relative amount connecting the displacement (direct distance from the starting position to the final position of the cell) and the total path length covered by the cell. After seeding the HT1080 cells threedimensionally inside the hydrogel, we observed through LSCM the cell migration to be significantly affected by the pore size in the three different regions of the hydrogel in the control device (Figure 3). 


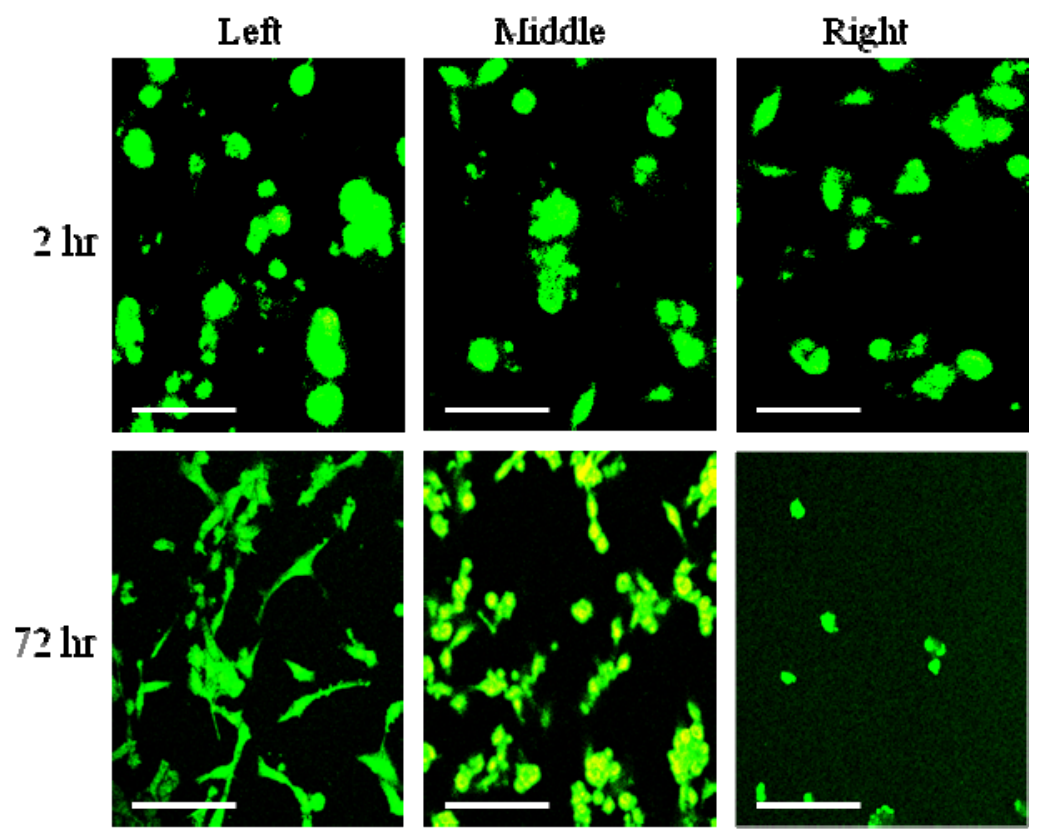

Figure 3 Cells distribution through the porous gradient in the control device where the left and right reservoirs were supplied with complete medium. Images were acquired after $2 \mathrm{hrs}$ and after $72 \mathrm{hrs}$. The scale bar denotes a length of $50 \mu \mathrm{m}$.

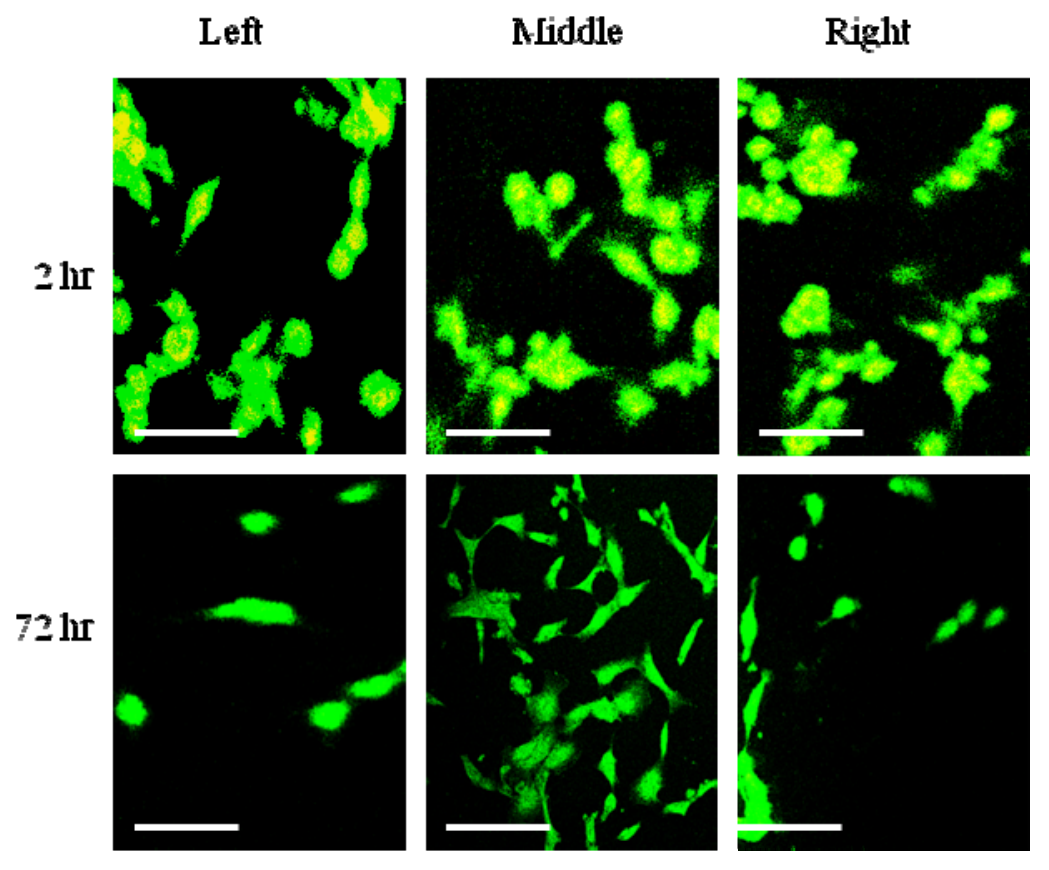

Figure 4 Cells distribution through the porous gradient in the chemotaxis device where the left reservoir was supplied with DMEM without serum, while the right reservoir was supplied with only FBS serum as chemoattractant material. Images were acquired after 2 hrs and after $72 \mathrm{hrs}$. The scale bar denotes a length of $50 \mu \mathrm{m}$. 
Most cells were observed to migrate to the left region that contains the largest pore especially after three days, whereas few cells remained in the right region due to the small pore size in the Gtn-HPA/CMC- Tyr structure. The cells appeared to remain spherical in shape with little spreading in both middle and right regions. When chemoattract was present in the right chamber, Figure 4 shows that the HT1080 cells migrated to the middle region as some of them preferred to migrate to the right where the chemoattractant concentration is large, while others preferred to migrate to the left where the larger pores offer lower mobility resistance and high growth level. Most notably, the morphology of the cells located in the middle gradient regions gradually changed from spherical to shapes that are well-spread.

\section{CONCLUSION}

We successfully demonstrated a microfluidic device that contains a porous gradients to study the monitoring the chemotactic responding of HT1080 cancer cells. Our study confirmed the validity of using in situ porous hydrogels as a construction material for a microchemotaxis device and showed the potential of the hydrogel with tunable porosity based microfluidic device in biological experiments.

\section{ACKNOWLEDGEMENTS}

The authors would like to acknowledge support from the Staff Member Research Fund from the Faculty of Engineering, Monash University. This work was performed in part at the Melbourne Centre for Nanofabrication (MCN).

\section{REFERENCES}

[1] Li, S., J.L. Guan, and S. Chien, "Biochemistry and biomechanics of cell motility", Annu. Rev. Biomed. Eng. 7, 105-150 (2005).

[2] Boyden, S., "The chemotactic effect of mixtures of antibody and antigen on polymorphonuclear leucocytes", The Journal of experimental medicine. 115, 453-466 (1962).

[3] Wells, C.M. and A.J. Ridley, "Analysis of cell migration", Clifton, N.J., 294, 31-41 (2005).

[4] Servant, G., et al., "Polarization of chemoattractant receptor signaling during neutrophil chemotaxis", Science. 287(5455), 1037-1040 (2000).

[5] Lee KY and M. DJ., "Hydrogels for tissue engineering", Chem Rev. 101, 1869-1879 (2001).

[6] Lee, B.H., et al.," In-situ injectable physically and chemically gelling NIPAAm-based copolymer system for embolization". Biomacromolecules, 7(6), 2059-2064 (2006).

[7] Liu, Y.Y., Y.H. Shao, and J. Lu,"Preparation, properties and controlled release behaviors of pH-induced thermosensitive amphiphilic gels". Biomateriala, 27(21), 4016-4024 (2006). 